\title{
A definição de prioridade de investimento em saúde: uma análise a partir da participação dos atores na tomada de decisão
}

\author{
I ${ }^{1}$ Francisco de Assis Santos, ${ }^{2}$ Garibaldi Dantas Gurgel Júnior, ${ }^{3}$ Idê Gomes Dantas \\ Gurgel, ${ }^{4}$ Hélder Freire Pacheco, ${ }^{5}$ Adriana Falangola Benjamin Bezerra I
}

\begin{abstract}
Os problemas de saúde possuem contornos próprios e a ação governamental para a elaboração de políticas públicas de saúde pode ser construída a partir da contribuição de diversos atores para a tomada de decisão. $\mathrm{O}$ estudo teve como objetivo analisar o processo de tomada de decisão sobre as prioridades de investimento a partir da percepção de diversos atores. Como método, é do tipo exploratório de corte transversal, com a utilização de abordagem qualitativa para coleta e análise de dados. Foram entrevistados 18 atores e realizados dois grupos focais, com gestores e membros do Conselho Estadual de Saúde. Concluiuse que, na definição de prioridades para os rumos da política de saúde, no caso do ente federado estudado, os aspectos eleitorais suplantaram questôes técnicas e a negociação dentro dos espaços colegiados de governança do SUS. As características da federação brasileira e a formatação do aparelho de Estado na saúde favorecem o fortalecimento de pautas relacionadas ao ciclo eleitoral na agenda governamental e na elaboração de políticas públicas, preterindo evidências científicas e outros aspectos técnicos para a tomada de decisão.
\end{abstract}

> Key words: saúde pública; política de saúde; agenda de saúde; planejamento em saúde.

\author{
1 Grupo de Pesquisa em Saúde \\ Pública, Faculdade ASCES; \\ Faculdade de Medicina, \\ Universidade Federal de \\ Pernambuco. Caruaru-PE, \\ Brasil. Endereço eletrônico: \\ francisco.a.santos@hotmail.com \\ ${ }^{2}$ Núcleo de Estudos em Saúde \\ Coletiva, Fundação Oswaldo \\ Cruz. Recife-PE, Brasil. Endereço \\ eletrônico: gurgel@cpqam. \\ fiocruz.br \\ ${ }^{3}$ Núcleo de Estudos em Saúde \\ Coletiva, Fundação Oswaldo \\ Cruz. Recife-PE, Brasil. Endereço \\ eletrônico: ideg@cpqam. \\ fiocruz.br \\ ${ }^{4}$ Núcleo de Estudos em Saúde \\ Coletiva, Fundação Oswaldo \\ Cruz. Recife-PE, Brasil. Endereço \\ eletrônico: helder-pacheco@ \\ bol.com.br \\ ${ }^{5}$ Faculdade de Medicina, \\ Universidade Federal de \\ Pernambuco. Recife-PE, \\ Brasil. Endereço eletrônico: \\ afalangola@uol.com.br
}

Recebido em: 28/03/2014 Aprovado em: 02/10/2014 
O estudo das políticas públicas se insere, hoje, nos esforços para compreender o papel do Estado e sua forma de intervenção na sociedade. Jardim, Silva e Nharreluga (2009) acrescentam que isto significa observar as interaçôes entre o Estado e a sociedade, identificar as relações entre os atores e compreender a dinâmica da ação pública. É possível fazer uma análise de política, aprofundar questôes relacionadas ao poder dos diversos atores que participam do processo político, sobretudo na definição de prioridades, verificando-se a existência e percepção da pluralidade de atores político-institucionais que vêm influenciando a constituição das políticas de saúde.

Entende-se que o poder é construído a partir de múltiplas forças, que de maneira ideal atuam não apenas de cima para baixo, mas em todos os lados; verifica-se que, quando se fala em processo de políticas de saúde, existem algumas forças que atuam de maneira mais organizada na construção dessas políticas. Para Santos et al. (2009), os grupos de atores podem ser separados em função de seus interesses e poderes para participar do processo político, os quais são distribuídos em: população ou usuários; sujeitos ou profissionais (técnicos); e líderes. E, dependendo do processo, qualquer um destes grupos pode ser jogador de uma política de saúde.

Há autores que advogam a existência de, pelo menos, três grupos distintos que podem compor a construção da política pública: a gestão, a academia e os profissionais (técnicos). Estes três nichos possuem expectativas e comportamentos distintos, que não os impedem de buscar a atuação conjunta na construção de uma política de saúde (JANSEN et al., 2010).

Percebe-se que as relações de poder intergovernamental enraízam-se no conjunto das redes de políticas públicas no Sistema Único de Saúde (SUS) com redefinição do lócus de produção e tomam vulto na necessidade de compartilhar o processo de tomada de decisão nos espaços de governança. Um dos objetos importantes da análise de política de saúde no Brasil está relacionado ao entendimento do papel dos diversos atores envolvidos nos processos políticos.

Para Portela e Teixeira (2011), os problemas de saúde possuem contornos próprios, visto que: 1) as necessidades em saúde são urgentes; 2) geralmente são de amplo impacto social e político; 3) as intervenções devem ter um aspecto multidimensional, isto é, ser intersetoriais; e 4) as ações não podem sofrer 
descontinuidade, devem ser centradas nos princípios da universalidade e equidade.

Assim sendo, a tomada de decisão deve atender a uma série de critérios políticos, econômicos, culturais, científicos, epidemiológicos, e sociossanitários, tendo em vista que as intervenções no campo das políticas de saúde geralmente possuem um alto valor agregado e representam um grande custo para a sociedade. Acrescentese ainda que a demanda por serviços de saúde não é facilmente compreendida, em virtude do caráter simbólico e subjetivo que existe por trás de uma necessidade de saúde (CECÍLIO, 2009; FRANCO; MERHY, 2005). Os pesquisadores da área atuam discutindo e elaborando pesquisas no campo da Saúde Coletiva, no intuito de gerar evidências científicas que possam embasar a tomada de decisão para novas alternativas no sentido da prevenção das doenças, da promoção e da organização de um sistema equânime de saúde (BARRETO, 2003).

A tomada de decisão que orienta a oferta de serviços em saúde pode ser influenciada por várias questões, tais como fatores situacionais (eventos violentos ou mudança de governo), que são mais ou menos transitórios, e fatores estruturais (estrutura política, economia, sistemas sociais) que são características relativamente permanentes de determinado país; fatores culturais (valores da sociedade) e os fatores ambientais (TROSTLE; BRONFMAN, LANGER, 1999). Souza (2006) apresenta os três principais mecanismos para chamar a atenção dos decisores e formuladores de políticas públicas: (a) indicadores negativos referentes a determinado problema; (b) catástrofes naturais ou epidemias; e (c) feedback, ou informações que mostram as falhas da política e resultados medíocres. Pode-se acrescentar as pesquisas eleitorais como motivações para mudança de uma agenda política e tomada de decisão e a influência da opinião pública via sistemas de comunicação.

A autoridade sanitária do Estado de Pernambuco, a partir do ano de 2007, com a mudança de governo estadual decorrente do processo eleitoral, por meio da Secretaria de Saúde do Estado, passou a defender a ampliação da rede sob sua responsabilidade, com aumento do número de prestadores de serviços e leitos, construindo três hospitais de grande porte de referência na Região Metropolitana do Recife, 14 Unidades de Pronto-Atendimento (UPAs) e ampliação da rede conveniada ao SUS. Com essa expansão e mudança na estrutura de serviços, observa-se a entrada de novos atores e inserção de novos arranjos institucionais, que passaram a se responsabilizar pela execução das ações, qualificados como Organizaçôes Sociais, com mudança do modelo de gestão tradicional, baseado 
no comando e controle vertical estatal para contratualização de entes de natureza privada e regulação da demanda assistencial. Assim, além da rede própria estatal, houve incremento substancial de recursos e franca expansão do financiamento das ações do setor de saúde.

A análise da participação dos atores no processo permite compreender a política a partir da formulação e do jogo de interesse de quem de fato atuou. Em virtude da contemporaneidade dos fatos e importantes alterações da política de saúde em Pernambuco, principalmente entre 2007 e 2012, cabe analisar como ocorreu o processo de tomada de decisão sobre as prioridades de investimento e a percepção dos diversos atores sobre a construção da política de saúde, enfatizando os investimentos realizados na abertura de três grandes serviços hospitalares.

\section{Método}

O estudo foi do tipo exploratório de corte transversal, com a utilização de abordagem qualitativa para coleta e análise de dados, tendo sido realizado entre os anos de 2007 e 2012. A área estudada possui uma população de 8.796 .032 habitantes distribuídos em 184 municípios e um distrito estadual. Desde o ano de 2011, quando foi elaborado o último Plano Diretor de Regionalização (PDR), o Estado de Pernambuco foi dividido em 12 Regiōes de Saúde e quatro macrorregiōes (PERNAMBUCO, 2011).

Os atores que representam os municípios foram selecionados a partir daqueles que integravam as câmaras técnicas, e que participaram do curso de gestão regionalizada oferecido pela Fiocruz-PE, em parceria com a Secretaria Estadual de Saúde, que ocorreu nas quatro macrorregiōes do estado durante o ano de 2012. Os informantes da gestão estadual foram todos os secretários estaduais de Saúde do período de 2007 a 2012, assim como três secretários executivos que tiveram papel relevante na discussão da política de saúde em Pernambuco, indicados pelos gestores estaduais. Ainda houve a participação de técnicos do Ministério da Saúde e do Conselho Nacional dos Secretários Municipais de Saúde (Conasems), responsáveis por acompanhar a política do estado, e de dois importantes pesquisadores, especialistas da área de Planejamento e Gestão.

Foram realizados dois grupos focais: 1) o grupo focal dos gestores municipais teve a participação de dez secretários de Saúde que representavam todas as quatro macrorregiōes do estado; 2) o grupo focal do Conselho Estadual de Saúde teve 
oito participantes, quatro representantes de usuários e quatro de trabalhadores da saúde. Toda a coleta de dados ocorreu entre maio e dezembro de 2012. Para análise das falas, foi utilizada a técnica de condensação de significados de Kvale (1996).

Para o conhecimento da participação da população (comunidade em geral), foi realizada uma pesquisa qualitativa em base de dados secundários, foram observados relatórios dos seminários "Pernambuco Para Todos" (iniciativa do governo estadual de redesenhar o planejamento a partir de demandas da população), pesquisas de opinião sobre a saúde estadual e publicações correlatas.

Ao final, os grupos foram distribuídos em: a) população - comunidade em geral, representando aqueles que não participam de nenhum espaço institucional de discussão das políticas de saúde; b) sujeitos e profissionais (técnicos) - seis técnicos municipais, representando todas as macrorregiōes do estado, três técnicos da Secretaria Estadual de Saúde, dois gestores do Ministério da Saúde (Gestor MS), um do Conselho Nacional de Secretários Municipais de Saúde (Técnico Nacional) e, por fim, dois membros da academia (Pesquisador); c) líderes foram entrevistados: quatro secretários estaduais de Saúde (Gestor Estadual) do período de 2007 a 2012 e os grupos focais com gestores municipais (Grupo Focal GM) e com o Conselho Estadual de Saúde (Grupo Focal CES). Ao total, foram contabilizados 18 entrevistas e dois grupos focais, realizados entre maio e outubro de 2012. O modelo de Eden (1996), adaptado por Santos et al. (2011), guiou a análise dos dados (figura 1).

Figura 1. Organização de categorias de indivíduos para análise de atores, segundo o interesse e o poder.

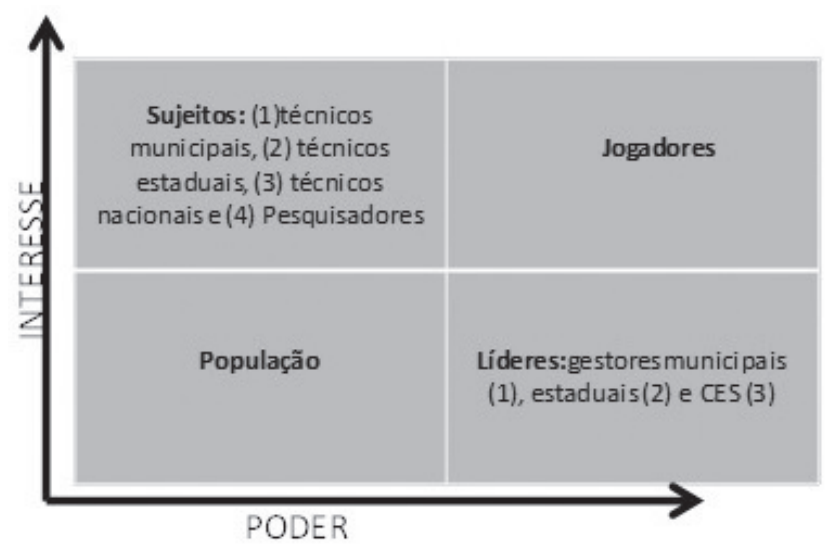

Fonte: Santos et al. (2011). 
Os resultados são apresentados a partir de trechos das entrevistas realizadas e apresentação da movimentação dos atores, enquanto jogadores, no processo político e na tomada de decisão. $\mathrm{O}$ estudo faz parte da pesquisa intitulada $O$ impacto das Reformas Estruturais no SUS: uma análise compreensiva sobre gasto público, acesso e performance da assistência à saúde de 2006 a 2011, aprovada pelo Comitê de Ética em Pesquisa do Cento de Pesquisa Aggeu Magalhães, sob o número CAEE 0037.0.095.408-11, estando conforme a Resolução no 466/13 do Conselho Nacional de Saúde.

\section{Resultado}

As características legais do SUS garantem a participação social e a criação de comissōes intergestores, no sentido de favorecer a distribuição do poder de decisão e governança do sistema de saúde. Em Pernambuco, há divergência entre os atores pesquisados, no que diz respeito à tomada de decisão sobre investimentos e disposição de serviços no território, quando comparamos as falas dos gestores estaduais e municipais:

Diagnosticamos também em cada regional e fomos para o contato direto, recebemos os técnicos das secretarias municipais e com os nossos técnicos e definindo um plano de ação de três anos, e assim foi feito. [...] Todas as nossas ações foram analisadas, subsidiadas e homologadas pela sociedade, seja nos pequenos municípios, nos grandes municípios. (Gestor Estadual 3).

[...] do ponto de vista formal, eu diria que todas as instâncias então sendo respeitadas, o conselho aprovou o plano e construiu junto com a gente o plano estadual de saúde [...]. (Gestor Estadual 3).

Os técnicos municipais, gestores municipais e Conselho Estadual de Saúde apontam a característica centralizadora por parte do Estado, na tomada de decisão:

Não passou nem pela porta pra a gente aprovar, já foi construído lá fora [...] Muitas vezes a gente percebe na questão governamental, que a coisa não é como o usuário, ou como a gente quer. Já vem pronta, de cima pra baixo, em efeito cascata. O governo faz, não comunica as entidades, não estimula a participação. (Grupo Focal CES).

“[...] e eu acho que o Estado ele faz alguma coisa quando é conveniente a ele. [...] ele faz quando lhe é conveniente e quando lhe dá resultado. (Grupo Focal SMS).

[...] essa opção do estado investir em três grandes hospitais na entrada da capital, é uma opção, mas que pra mim não é a melhor opção, eu tô falando dentro de uma perspectiva da economia da saúde, em que a gente sabe que o recurso é limitado, e que consequentemente você tem que fazer escolha, e em se tratando de dinheiro público, boas escolhas, as melhores escolhas... (Pesquisador 1). 
Aqui a gente sofre muito com a aglomeração de serviços onde já existe, eu sou muito

crítico ao Estado de Pernambuco porque eu não enxergo uma gestão com um olhar técnico para as necessidades de cada região, eu vejo muito a questão da política, questão do jogo de interesses. (Técnico Municipal 2).

A administração desses serviços passou a ser realizada por instituições privadas sem fins lucrativos. Ao se analisar a fala dos atores, observa-se semelhança quanto à discussão referente à definição de prioridade de investimentos, previamente apresentada. A postura da gestão estadual é favorável ao modelo, enquanto os gestores municipais, conselhos de saúde e técnicos são reticentes quanto aos possíveis impactos. Há queixas sobre o modo de condução dos processos, com forte concentração de poder de decisão nas mãos da gestão estadual.

[...] questão das organizações sociais dentro da saúde e se a gente for buscar isso, essa discussão e o resultado disso na Europa, a gente vê que houve uma época que as PPSs estavam em alta, mas hoje está [sic] no processo de declínio, então porque [sic] nós estamos copiando uma coisa que comprovadamente não funciona em outro país. (Grupo Focal CES).

[...] Então eu acho que foi simplista a posição do estado de Pernambuco em fazer opção pelas OSSs. E aí vem essa coisa mesmo. É dizer que nós somos incompetentes como gestores [...]. (Grupo Focal GM).

A própria gestão estadual, mais de três anos após a inauguração do primeiro serviço administrado por organizações socias da saúde (OSSs), não possuía evidências concretas quanto às vantagens financeiras do modelo implantado:

[...] A comparação (serviços próprios e administrados por OSS), nós temos indicadores custo, e como a gente em Pernambuco não tem ainda o custo por procedimento, não temos ainda, mas vamos ter, usamos ainda os indicadores das tabelas, então as nossas unidades administradas por OSS estão rodando duas vezes e meia a três vezes a tabela SUS, em São Paulo é mais quatro, cinco vezes. Os hospitais próprios são na base de 5 a 6 vezes a tabela do SUS, mas isto ainda não está fechado, contratamos uma consultoria para avaliar" [...] (Gestor Estadual 2).

Pode ser constatado que a agenda foi influenciada por critérios políticoeleitorais, advindos da campanha eleitoral e que moldaram a construção do processo político, como demonstrado nas falas a seguir:

A definição foi priorizar como o governador sempre fez a construção de três hospitais, como ele tinha prometido na campanha. (Gestor Estadual 1).

[...] nós construímos três hospitais, que foi um compromisso do governador Eduardo Campos, que cumpriu integralmente, [...] até porque eles estão distribuídos geograficamente em pontos estratégicos na região metropolitana. (Gestor Estadual 3). 
[...] mas isso fez parte do programa de governo, que foi aprovado pela população, $90 \%$ do eleitorado, então como programa de governo estava legítimo [sic] a definição de priorização na construção desses dispositivos e desses equipamentos [...]. (Técnico Estadual 3).

[...] muitos vazios assistenciais no estado, e uma demanda ainda muito grande pra região metropolitana. Essa opção do estado de investir em três grandes hospitais na entrada da capital é uma opção, mas que pra mim não é a melhor opção, eu to [sic] falando dentro de uma perspectiva da economia da saúde... (Pesquisador 1).

Observando-se os depoimentos dos atores envolvidos com a tomada de decisão sobre a política estadual de saúde em Pernambuco, destaca-se que há participação de gestores e técnicos estaduais diretamente sobre o jogo político. A população participa indiretamente do processo, ao legitimar a plataforma de governo defendida ainda no ano de 2006 pelo grupo vencedor do processo eleitoral e reeleito em 2010, com uma das maiores aprovaçóes em todo o Brasil.

\section{Figura 2. Comportamento dos atores no processo de tomada de decisão de} prioridades de investimentos em Pernambuco.

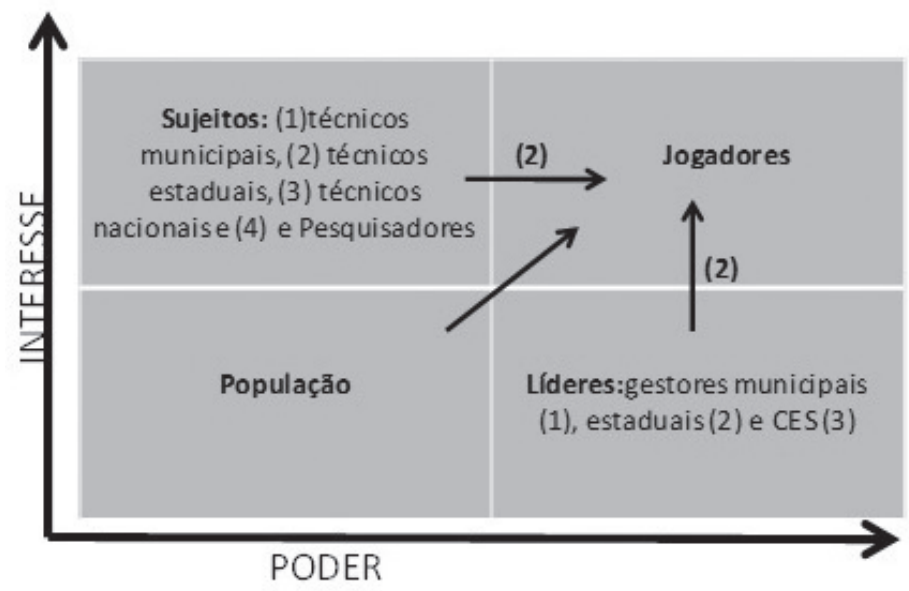

Fonte: Santos et al. (2011).

\section{Discussão}

A formulação de políticas no SUS é compartilhada entre os gestores no âmbito das comissóes intergestores e com outros atores sociais, por meio dos conselhos de saúde. Nesses espaços de governança deveriam ser aprovadas as diretrizes de política de saúde, sobretudo a prioridade de investimentos. Para Machado, Lima 
e Baptista (2011), a contribuição de diversos atores no processo de construção da

política não acontece de forma homogênea entre os diversos temas da política. Isso corrobora os dados da pesquisa, demonstrando que não há consenso sobre o compartilhamento de poder durante a elaboração da política de expansão dos serviços de saúde e a transferência da gestão para OSS.

A partir do fato de que a participação no processo de elaboração da política e a tomada de decisões ficam restritas a um pequeno grupo de atores, há possibilidade de falhas relevantes durante o período em que a política é colocada em prática, problemas que podem não ser evidentes, mas posteriormente passam a surgir, tais como: baixa adesão por parte dos profissionais, intermitência do financiamento e pouca mudança nos indicadores de saúde no longo prazo (SANTOS et al., 2011). Por isso, o conhecimento do processo de tomada de decisão por meio da análise de política, considerando a participação dos atores, pode ter caráter prescritivo ou prospectivo, desde que de antemão se busque entender quem efetivamente contribuiu para a construção da política.

A autoridade sanitária de Pernambuco fez a escolha por mudar o modelo de gestão sem evidências científicas claras sobre os reais impactos econômicos e sanitários sobre o sistema de saúde. Isto vai de encontro ao que Macintyre (2012) chama a atenção como principal evidência analisada por parte dos políticos e dos formuladores de políticas públicas, que é o retorno financeiro das ações e serviços implantados no território. Pode-se observar que a literatura brasileira, e até a internacional, ainda não apresentam resultados que possam ser utilizados como referência para a implementação deste modelo de gestão privada no SUS em Pernambuco (PAHIM, 2009).

Critérios técnico-científicos foram preteridos para a adoção do novo modelo de gestão em Pernambuco. Para Elliot e Popay (2000), a influência direta da utilização de evidências em estudos políticos depende de fatores como: limitação financeira, mudança na equipe e experiências pessoais dos gestores. Geralmente, as pesquisas têm influência indireta sobre a política, colocando em pauta algumas discussões para o debate e mediando o diálogo entre os provedores e usuários dos serviços de saúde.

Pode-se afirmar que, em condições ideais, depois que o problema é definido, buscam-se as evidências, ou seja, coletar dados que podem ajudar a identificar importantes características do problema de política em estudo e como ele pode 
ser resolvido ou atenuado. Com isso, a tomada de decisão pode ser definida por critérios: científicos, técnicos, econômicos, políticos ou sociais (PETTICREW et al., 2004). No mundo real, um gestor raramente terá tempo para um esforço de pesquisa dentro de padrões de elevado rigor acadêmico (CHURCH et al., 2002). Por essa razão, a formulação de programas de governo tem tido baixa mobilização desses recursos, porém sobejamente lastreados em pesquisa de marketing políticoeleitoral para formulação do discurso e programas de governo.

No Brasil, convive-se com um modelo eleitoral que impõe eleições bianuais (municipais, intercaladas com estaduais e federais), uma série de coligações e acordos que potencializam a influência político-eleitoral na definição de prioridades, pois o horizonte de tempo é curto, e os cenários políticos, por falta de bases ideológicas sólidas, são mutáveis e permeados por interesses clientelísticos, patrimonialistas e excludentes de boa parte da sociedade, levandose em consideração a estrutura do aparelho de Estado na saúde.

O reflexo do modelo eleitoral sobre as políticas de saúde pode ser observado em estudo como o de Novaes e Matos (2010), que apontaram, em pesquisa de âmbito nacional, o impacto que a finalidade da reeleição e da intenção de se ter o sucessor político provoca sobre os gastos em saúde, isto é, quanto maior a intenção de se manter no poder, mais vultosos são os investimentos. Tomar decisões centradas em promessas de campanha pode não atender a critérios que contemplem a economicidade e as reais necessidades da sociedade. Como demonstrado por Scheffer e Bahia (2011), há grupos que vêm expandindo suas doaçôes durante o período eleitoral e, portanto, podem influenciar os rumos das políticas públicas. Deste modo, na realidade brasileira, há elevada dependência dos atores e do contexto político-eleitoral e partidário para que sejam definidas as prioridades de investimentos, mesmo que em detrimento de ações que tenham uma sustentação técnica ou forte apoio social.

Besley e Preston (2007) apontam que, mesmo em países onde os partidos políticos são consolidados e possuem plataformas ideológicas firmes, pode haver mudanças em determinadas tendências nas promessas políticas com o intuito de vencer as eleiçôes. A depender das circunstâncias, as prioridades podem ser guiadas por interesses político-eleitorais. Por conseguinte, mesmo sem um amparo técnico-científico definido, passam a fazer parte do programa de governo que será executado durante o mandato para o qual o governante foi escolhido. Sabatier 
(2007) é um dos autores que demonstram a força que uma promessa de campanha tem na moldura do processo político, fato que também ocorre em Pernambuco.

A definição da prioridade de investimento, com o enfoque, por exemplo, na construção de novos hospitais no entorno da região metropolitana da capital, reconhecida por possuir grande concentração de serviços médico-hospitalares (SANTOS et al., 2009), ocorreu antes mesmo de qualquer consulta ao Conselho Estadual de Saúde ou Conselho de Secretários Municipais de Saúde, ou outra entidade colegiada do SUS. Na verdade, foi uma promessa durante a campanha ao governo estadual em 2007 e, após a eleição do governador, passou a ser a promessa de campanha mais lembrada pela sociedade (CREMEPE, 2007). Para os gestores da saúde, a relevância principal ainda é dada pela opinião pública e seu impacto sobre os aspectos políticos e as tomadas de decisões (PETTICREW et al., 2004), pois uma demanda urgente pode preceder qualquer evidência científica; por conseguinte, antes de procurar evidências, os gestores definem estritamente a questão política a ser examinada (CHURCH et al., 2002).

Em Pernambuco, houve prioridade para assistência em caráter hospitalar de alta complexidade e alto custo, via parcerias público-privadas, a partir da implementação de mais três unidades à rede estadual de saúde. A tomada de decisão, com pouca participação direta de atores tradicionalmente relevantes na construção do processo político, pode ser consequência não apenas do modelo político-eleitoral brasileiro, mas também da organização do país em federação e da própria estrutura e dinâmica das autoridades sanitárias do país, ocupadas por elevado número de cargos comissionados. Esse fato reflete a forte influência do processo eleitoral na provisão e preenchimento dos empregos públicos, em detrimento do estamento burocrático baseado em carreiras de Estado, com predomínio de técnicos nessas estruturas de elaboração de políticas. Estudos apontam que a organização político-administrativa em federação pode favorecer o beneficiamento político sem a utilização de critérios técnicos para a destinação de recursos, fato comprovado por Esselment (2012), no Canadá, e por Timmons e Broid (2013), no México, onde os interesses político-partidários são apontados como os principais motivadores para investimentos em determinadas ações realizadas em municípios ou regiōes.

Esta pesquisa apresenta que houve participação direta dos gestores estaduais, técnicos, e indireta da população. A opinião da população pode ser mensurada a 
partir de pesquisas de satisfação quanto ao serviço de saúde, como a realizada por Moimaz et al. (2010) em cinco cidades de São Paulo. Contudo, a participação da população na definição dos rumos da política pode ser contabilizada como exemplo de participação indireta nos rumos da política de saúde de Pernambuco, devido à legitimidade que foi ofertada às propostas, o que contribuiu para o Executivo estadual ser avaliado como o melhor gestor, em todo o Brasil (CNI, 2013). A sociedade participou de maneira tangencial da definição dos rumos da política, sem utilizar-se dos espaços formais de governança, como os Conselhos de Saúde. Church et al. (2002) esclarecem que a participação da sociedade na discussão da política é feita em diversos patamares, dependendo da distância para o topo: quanto mais distante, menor a contribuição. Os autores sugerem que a efetiva participação na tomada de decisão pode ocorrer quando há espaços como comitês ou juntas de planejamento que contam com a participação de diversos atores.

A tomada de decisões baseada em questôes eleitorais, sem levar em conta a evidência científica ocorre porque, em primeiro lugar, enfatiza-se que o estudo científico é apenas uma entrada (input) dentre muitos outros elementos igualmente legítimos de serem considerados pelos decisores, como: as demandas sociais, político-eleitorais e econômicas. Tal fato limita o consenso sobre a possibilidade e a obrigação dos políticos e programadores para considerar os resultados de investigações na tomada de decisōes. Em segundo lugar, mesmo os resultados mais atraentes de pesquisa de alta qualidade devem ser avaliados em termos de custos financeiros e político-eleitorais, antes que possam ser considerados como base para uma política ou programa (TROSTLE; BRONFMAN; LANGER, 1999).

Em Pernambuco, constata-se que os colegiados intergestores e o Conselho Estadual de Saúde tiveram pouca participação na definição das prioridades de investimentos para o setor saúde, a população atuou indiretamente e houve afastamento da Academia na construção das políticas públicas. $\mathrm{O}$ cenário se contrapõe às recomendações de priorizar as evidências científicas, utilizando critérios técnicos no desenvolvimento e implementação de políticas de estado e programas governamentais.

\section{Considerações finais}

A gestão estadual da saúde em Pernambuco desencadeou profundas mudanças na política de saúde, desde a abertura de novos serviços, até a implementação 
de um novo modelo de gestão. Contudo, as diferentes visões sobre o processo

de tomada de decisão sobre investimentos evidenciam a influência dos ciclos eleitorais nas políticas públicas.

Fatores levantados neste artigo apontam dificuldades no processo de governança e a necessidade de definir prioridades que contemplem critérios epidemiológicos, sociais e econômicos, para garantir maior efetividade da política de saúde. Principalmente, considerando como se comportam as forças políticas e os agentes públicos, sobretudo aqueles associados a fortes interesses eleitorais, que por diversas vezes desconhecem critérios que apontam para a relevância técnica e a sustentabilidade econômica das propostas de intervenção do Estado. Por isso, ainda há um longo caminho a ser percorrido para que as prioridades possam ser construídas para além do horizonte de uma campanha política.

O estudo aponta a necessidade de legitimar tais decisóes nos espaços de governança do SUS, principalmente naqueles com maior participação da sociedade. Aponta também a necessidade de fortalecer as decisões colegiadas, aprimorando os mecanismos que buscam aproximar a evidência científica da política de saúde (política baseada em evidência) e, com isso, construir uma discussão democrática, baseada em informações técnicas, que seja mobilizadora da opinião pública no longo prazo. ${ }^{1}$

\section{Referências}

BARRETO, M. L. A pesquisa em saúde coletiva. Cad. Saúde Pública, Rio de Janeiro, v. 19, n. 2, p. 354-355, 2003.

BESLEY, T.; PRESTON, I. Electoral bias and policy choice: theory and evidence. The Quarterly Journal of Economics, Massachusetts, v. 122, n. 4, p. 1473-1510, 2007.

CECILIO, L. C. O. As necessidades de saúde como conceito estruturante na luta pela Integralidade e Equidade na atenção em saúde. In: PINHEIRO, R.; MATTOS, R. A. (Orgs.). Os sentidos da integralidade na atenção e no cuidado à saúde. Rio de Janeiro: Cepesc, 2009. p. 117-130.

CHURCH, J. et al. Citizen participation in health decision-making: past experience and future prospects. Journal of Public Health Policy, Londres, v. 23, n. 1, p. 12-32, 2002.

CNI-IBOPE. Avaliação de Governo. 2013. Disponível em: <http://arquivos. portaldaindustria.com.br/app/conteudo_24/2013/12/13/52/20131213112444335098o. pdf>. Acesso em: 27 dez. 2013. 
CREMEPE. 100 dias: promessa de construir hospitais é a mais lembrada. 2007. Disponível em: <http://www.cremepe.org.br/leitorClipping.php?cd_clipping=7929>. Acesso em: 2 jan. 2014

EDEN, C. The Stakeholder/Collaborator Strategy Workshop. In: HUXHAM, C. (Ed.) Creating collaborative advantage. London: Sage, 1996. p. 44-56.

ELLIOTT, H.; POPAY, J. How are policy makers using evidence? Models of research utilisation and local NHS policy making. Journal of Epidemiology and Community Health, v. 54, n. 6, p. 461-812, 2000.

ESSELMENT, A. L. A little help from my friends: The Partisan Factor and intergovernmental negotiations in Canada. Publius, London, v. 43, n. 4, p. 701-727, 2012.

FRANCO, T. B.; MERHY, E. E. Produção imaginária da demanda. In: PINHEIRO, R.; MATTOS, R. A. (Orgs.) Construção social da demanda: Direito à saúde, trabalho em equipe, participação e espaços públicos. Rio de Janeiro: Cepesc, 2005. p. 183-195.

JANSEN, M. W. et al. Public health: disconnections between policy, practice and research. Health Research Policy and Systems, London, v. 8, n. 1, p. 37, 2010.

JARDIM, J. M.; SILVA, S. C. de A; NHARRELUGA, R. S. Análise das políticas públicas: uma abordagem em direção às políticas públicas de informação. Perspectivas em Ciência da Informação. Belo Horizonte, v. 14, n. 1, 2009. Disponível em: < http://www.scielo.br/pdf/ pci/v14n1/v14n1a02.pdf >. Acesso em: 17 set. 2012.

KVALE, S. Interviews: an introduction to qualitative research interviewing. Thousand Oaks: Sage, 1996. 326 p.

MACHADO, C. V.; LIMA, L. D.; BAPTISTA, T. W. F. Princípios organizativos e instâncias de gestão do SUS. In: GONDIM, R; GRABOIS, V; MENDES, V. (Orgs.). Qualificação dos Gestores do SUS. Rio de Janeiro: Fiocruz/ENSP/EAD, 2011. p. 47-72. Disponível em: http://www5.ensp.fiocruz.br/biblioteca/dados/txt_339793983.pdf. Acesso em: 19 set. 2012.

MOIMAZ, S. A. S. et al. Satisfação e percepção do usuário do SUS sobre o serviço público de saúde. Physis, Rio de Janeiro, v. 20, n. 4, p. 1419-1440, 2010.

MACINTYRE, S. Evidence in the development of health policy. Public Health, v. 126, n. 3, p. 217-219, 2012.

NOVAES, L.; MATTOS, E. O efeito da intenção de reeleição sobre gastos em saúde: uma análise com base no modelo de reputação política. Revista de Economia Política, São Paulo, v. 30, n. 117, p. 140-158, 2010.

PAHIM, M. L. L. Organizaçôes Sociais de Saúde do estado de São Paulo: inserção privada no SUS e gestão financeira do modelo pela Secretaria de Estado da Saúde. 2009. 250 p. Tese (Doutorado em Medicina Preventiva) - Faculdade de Medicina, Universidade de São Paulo, São Paulo, 2009. 
PERNAMBUCO. Secretaria de Saúde. Plano Diretor de Regionalização do SUS/PE.

Recife, 2011.

PETTICREW, M. et al. Evidence for public health policy on inequalities: the reality according to policymakers. Journal of Epidemiology and Community Health, v. 58, n. 10, p. 811-821, 2004.

PORTELA, L. E.; TEIXEIRA C. F. Epidemiologia e gestão de serviços de saúde. In: BARRETO, M. L.; ALMEIDA FILHO, N. Epidemiologia \& Saúde: fundamentos, métodos e aplicaçóes. Rio de Janeiro: Guanabara Koogan, 2011. p. 622-630.

SABATIER, P. A. Theories of the policy process. 2. ed. Boulder: Westview Press, 2007. 352 p.

SANTOS, M. O. S. et al. O polo médico do Recife. In: AUGUSTO, L. G. S. (Orgs.). Saúde do trabalhador e sustentabilidade do desenvolvimento humano local. Recife: Editora Universitária da UFPE, 2009. p. 219-245.

SANTOS, F. A. S. et al. Política de práticas integrativas em Recife: análise da participação dos atores. Revista de Saúde Pública, São Paulo, v. 45, n. 6, p. 1154, 2011.

SCHEFFER, M.; BAHIA, L. Representação política e interesses particulares na saúde: o caso do financiamento de campanhas eleitorais pelas empresas de planos de saúde privados no Brasil. Interface, Botucatu, v. 15, n. 38, p. 947-956, 2011.

SOUZA, C. Políticas públicas: uma revisão de literatura. Sociologias, Porto Alegre, v. 8, n. 16, p. 20-45, 2006.

TIMMONS, J. F.; BROID, D. The political economyof municipal transfers: evidence from Mexico. Publius, London, v. 43, n. 4, p. 551-579, 2013.

TROSTLE, J.; BRONFMAN, M.; LANGER, A. How do researchers influence decisionmakers? Case studies of Mexican policies. Health Policy and Planning, v. 14, n. 2, p. 103114, 1999.

\section{Nota}

${ }^{1}$ F. de A. Santos e G. D. Gurgel Júnior participaram da concepção, análise e interpretação dos dados, redação do artigo e aprovação da versão a ser publicada. I. G. D. Gurgel participou da elaboração do artigo e revisão crítica do conteúdo intelectual. H. F. Pacheco e A. F. B. Bezerra realizaram a revisão crítica do conteúdo intelectual e aprovaram a versão final a ser publicada. 
The definition of investment priorities in health: an analysis from the participation of stakeholders in decision-making

Health problems have their own contours and governmental action for the development of public health policies, can be built from the contribution of various actors for decisionmaking. This study aimed to analyze how was the process of making decisions about investment priorities and perceptions of the various actors on the construction of health policy in Pernambuco state, Brazil. As a method, it is an exploratory cross-sectional study, using qualitative approaches to collect and analyze data. Eighteen actors conducting two focus groups with managers and members of the state board of health were interviewed. It concludes that in setting priorities for the future of health policy in Pernambuco, electoral aspects overcame technical issues and negotiation within the collegiate governance spaces SUS. The characteristics of the Brazilian federation and State apparatus formatting favor strengthening health-related election cycle guidelines on the government agenda and the development of public policies, postponing scientific evidence and other technical aspects for decision-making.

> Key words: public health; health policy; agenda planning; health planning. 